\title{
Effect of mono and diglyceride of medium chain fatty acid on the stability of flavour emulsion
}

\author{
${ }^{1}$ Jadhav, H., ${ }^{2}$ Waghmare, J. and ${ }^{1 *}$ Annapure, U. \\ ${ }^{I}$ Department of Food Engineering and Technology, Institute of Chemical Technology, Matunga, Mumbai- \\ 400019 \\ ${ }^{2}$ Department of Oils, Oleochemicals and Surfactant Technology Institute of Chemical Technology, Matunga, \\ Mumbai-400019
}

\begin{abstract}
Article history:
Received: 16 November 2020

Received in revised form: 16 December 2020

Accepted: 16 February 2021

Available Online: 14 March 2021
\end{abstract}

\section{Keywords:}

Flavour emulsion,

Monocaprylin,

Dicaprylin,

Orange peel,

Homogenizer,

Emulsion stability

DOI:

https://doi.org/10.26656/fr.2017.5(2).589

\begin{abstract}
Flavour emulsion is used in a wide range of food products including carbonated beverages, dairy products, confectionaries, and bakery products. Among its food applications are extensive uses in bakery products due to its high heat resistant properties. In flavour emulsion flavour oil is suspended in water phase and this water phase retains flavour even if such flavour emulsion is used for high temperature food application like baking. But flavour extracts are made by suspending flavour oil in alcohol. So, such flavour extracts are not suitable for high temperature food processing, since alcohol evaporates at high temperature. Thus, flavour emulsion is much better than flavour extracts. The stability of flavour emulsion depends on the emulsifier used in making flavour emulsion. Monocaprylin and dicaprylin are mono and diglyceride of mediumchain fatty acids having very good emulsifying properties. The stability of flavour emulsion can be improved by making use of mono and diglyceride as an emulsifier. The present study focused on the development of stable flavour emulsion from fruit waste using mono and diglyceride of medium-chain fatty acid as an emulsifier. The stable flavour emulsion is made from orange oil, water and emulsifier using ultra high-pressure homogenizer with $25 \mathrm{MPa}$ pressure. Emulsifier mono and dicaprylin were synthesized by the esterification reaction between glycerol and caprylic acid. The effect of various combination of mono and dicaprylin is studied on the formation of stable flavour emulsion. The higher monocaprylin content in the emulsifier combination showed excellent results and a good synergistic effect on the stability of the emulsion. The combination of mono: diglyceride which resulted in the formation of stable flavour emulsion is $80: 20$ at $10 \%$ concentration, 2 passes and $25 \mathrm{mPa}$ pressure in a high-pressure homogenizer. The stability of flavour emulsion was investigated using a storage study. The emulsion was found to be stable for 30 days at ambient temperature $\left(25^{\circ} \mathrm{C}\right)$ and refrigeration temperature $\left(4^{\circ} \mathrm{C}\right)$ but showed low stability at $50^{\circ} \mathrm{C}$.
\end{abstract}

\section{Introduction}

An emulsion is a mixture of two immiscible liquids containing a continuous phase and dispersed phase. Mono and Diglyceride are used as an emulsifier in various food application. They account for about $70 \%$ of world food emulsifier production and hence considered as an important class of food emulsifiers (Norn, 2015). When one position of glycerol is esterified with fatty acid it forms monoglyceride. Monoglyceride contains two hydroxyl group. In diglyceride two positions of glycerol are esterified, hence diglyceride contains one hydroxyl group. Depending on the hydroxyl group the lipophilicity/hydrophilicity is decided. Since diglyceride contains only one hydroxyl group it is more lipophilic whereas monoglyceride which contains two hydroxyl group is more hydrophilic. This affects their emulsification activity. Hence, they are used in combination. The emulsification effect of the combination of mono and diglyceride will be more as compared to their effect as a single emulsifier (Nicholson and Marangoni, 2019). Jensen et al. (1961) reported monoglyceride content in ice-cream as an emulsifier in the range of 20.6 to $93.5 \%$. Monoglyceride gave a smooth texture and good emulsifying properties to icecream. 20 different sample of ice-cream were collected and were investigated for emulsifier content in it. Jenson 
et al. (1961) report a higher percentage of monoglyceride which is used as an emulsifier. Monoglyceride and diglyceride which are used as an emulsifier are mostly containing long-chain fatty acid esterified to the glycerol backbone. This includes oleic, linoleic acid (Jensen et al., 1961). Hasan et al. (2014) investigated the formation of a stable microemulsion of oil in water using mono and diglyceride of medium-chain fatty acid along with polyoxyethylene-25-glyceryl trioleate. Baer et al. (1997) investigated the effect of emulsifier lecithin on the body and texture of $2 \%$ fat containing ice-cream. The use of an emulsifier also results in good consistency of icecream and a reduction in time required for whipping. Loi et al. (2019) studied the effect of various combination of mono as well as diglyceride of saturated and unsaturated fatty acid on protein stabilized model emulsions. More monoglyceride content in the emulsifier mixture showed a good result is achieving small droplet sizes. Food industries across the globe make use of emulsifiers for making a stable emulsion. Processed food products such as ice-creams, sauces, carbonated beverages, cookies, hard candy, soft candy etc. make use of emulsifiers (Saifullah and Aziz, 2012). Flavours are used in food industries for a very long time. Flavours are extracted from plants, fruits etc. and they are stabilized using an emulsifier (Saifullah and Aziz, 2012). These flavour oils are extracted using solvent extraction, supercritical carbon dioxide, cold pressing, ultrasound-assisted extraction etc (Ropkins and Taylor, 1996; De Oliveira et al., 2013; Mazzutti et al., 2020; Ordóñez-Santos et al., 2020). These extracted flavour oils are used in the form of flavour emulsion or flavour extract in various processed foods like beverages, bakery etc. The benchmark of flavour emulsion in processed food is its stability during processing and storage. This benchmark can be achieved by using a good stabilizer and proper emulsification process during the production of flavour emulsion. In the present research, the higher stability of flavour emulsion is ensured by use on ultrahigh-pressure homogenizer. High pressure homogenizers are used to make a homogeneous mixture of continuous phase and dispersed phase. In homogenizers, mixture is forced to pass through a small opening at high velocity. This results in disruption of oil globules in to smaller ones. Now, these small oil globules get dispersed in the water phase as they pass through the homogenizer device. These oil globules have ability to form cluster after first stage of homogenization. To prevent the cluster formation the mixture is passed through second stage of homogenization. Thus, this process generates stable emulsion. The final size of the resulting emulsion is influenced by the viscosity of oil and water phases (Wilde, 2019). Membrane emulsification techniques are recent developments in emulsification processes that are widely used in foods, pharmaceuticals and cosmetic products. These techniques have good control over the stability of the final product (Spyropoulos et al., 2014; Osorio-Arias et al., 2021). Fruit waste such as peels is discarded after consumption. But these fruit peels are a potential source of various bioactive including flavouring compounds which are responsible for the aroma of fruits. Citrus fruits are well known for their natural flavour and mesmerizing aroma because of volatiles present which is highly concentrated in peels of citrus fruits. The citrus peels discarded after consumption of fruit can be utilized for extraction of these flavours and their utilization in processed foods (Wang et al., 2020). In the present work, mono and diglyceride of medium-chain fatty acid are used as an emulsifying agent for the formulation of a stable flavour emulsion from fruit waste using ultrahighpressure homogenizers.

\section{Materials and methods}

\subsection{Material}

Citrus peel oil was extracted in the lab, mono and digcaprylin were synthesized in the lab by esterification reaction, and distilled water was obtained from the distillation unit present in the lab.

\subsection{Collection of peels}

Orange peels were collected and dried to reduce the moisture content of orange peels to obtain maximum extraction. The orange peels were dried in a drying oven at $45^{\circ} \mathrm{C}$ for $5 \mathrm{hrs}$. The temperature was kept constant during the drying process.

\subsection{Extraction of oil from dried orange peels by cold pressing}

The cold pressing method is selected for the extraction of oil since this process gives more yield and retains the original properties of oil like fragrance, flavour, purity and the resulting product is free from impurities. The dried peels are milled into a paste using heavy stones. As it is ground the oil is separated from the peels. The oil is forced to move out from the press. The oil is then transferred to a filter cloth for filtering oil to remove impurities like part of the peel, fibre etc. After filtration, oil is decanted to remove any sediments present in the mixture. The obtained oil is further used for the application. Since no heat is used in this process, the natural volatiles aroma compound present in peels are not lost and a higher yield is obtained.

\subsection{Manufacturing of mono and dicaprylin}

The emulsifier used in the process was manufactured using an esterification reaction between glycerol and 
caprylic acid. The glycerol and caprylic acid on a molar basis are taken in a three-neck flask along with a catalyst. One neck of a reaction flask is connected to a condenser for condensing water vapour in order for the reaction to be conducted in a forward direction. The reaction was carried out at $180^{\circ} \mathrm{c}$ for 8 hours. The product obtained is a mixture of mono, di and tricaprylin. These are separated by fractionation. The reaction scheme is as followed:

Glycerol + Caprylic acid $\stackrel{\text { Catalyst } / \text { Temperature }}{\longrightarrow}$ Monocaprylin $+\mathrm{H}_{2} \mathrm{O}$ Glycerol + Monocaprylin $\stackrel{\text { Catalyst } / \text { Temperature }}{\longrightarrow}$ Dicaprylin $+\mathrm{H}_{2} \mathrm{O}$

The obtained monocaprylin and dicaprylin were used as an emulsifier for the development of flavour emulsion.

\subsection{Development of flavour emulsion}

Initially, the oil phase and water phase are prepared. The oil phase was added into the water phase along with an emulsifier. The mixture was then kept on a magnetic stirrer for mixing before the homogenization process. $70 \%$ of distilled water was mixed with $20 \%$ orange oil and $10 \%$ emulsifier. The premixed mixture is then homogenized using an ultra-high-pressure homogenizer. Approximately $30 \mathrm{~mL}$ mixture is taken in a glass beaker. This beaker is kept in a homogenizer and a collecting pipe is inserted in the beaker. $9 \mathrm{~mL}$ mixture is collected at a time, it is homogenized and the emulsified mixture is dropped in another beaker kept at an outlet. The effect of various passes and operating pressure was studied. Passes are varied from 1-4 and pressure up to $25 \mathrm{mPa}$.

\subsection{Analysis}

\subsubsection{Viscosity}

Viscosity was performed using Brookfield viscometer with LV 2 spindle for the best combination of mono: dicaprylin. Viscosity measurement was carried out in duplicate for all the sample of flavour emulsion. The average viscosity was expressed as apparent viscosity. Measurements of viscosity were observed with a shear rate up to $100 \mathrm{~s}^{-1}$. The shear rate of $100 \mathrm{~s}^{-1}$ resembles processes that are commonly used in food industries like pouring, mixing, pumping, agitation during the preparation of food emulsions (Shama and Sherman, 1973). Viscosity was measured before and after homogenization, to clearly understand the effect of the homogenization process on the flowability of emulsion.

\subsection{2 $\mathrm{pH}$}

The $\mathrm{pH}$ of all the flavour emulsion was measured using a $\mathrm{pH}$ meter at room temperature.

\subsubsection{Creaming index}

The creaming index was determined by the visual observation technique (McClements, 2007). All the samples of flavour emulsion were poured into glass bottles and these glass bottles were closed with a tight lid; the glass bottles were stirred mildly to ensure proper mixing and to make a homogeneous mixture and was stored at $30^{\circ} \mathrm{C}$. The creaming index refers to the ratio of the height of cream over the total height of emulsion when it is kept undisturbed.

\subsubsection{Stability study}

A stability study of all three flavour emulsion was carried out at room temperature, cold temperature $\left(4^{\circ} \mathrm{C}\right)$ and high temperature $\left(50^{\circ} \mathrm{C}\right)$ for 30 days. The sample was kept in a glass beaker with screw caps. These bottles were stored at three different temperatures i.e. room temperature $\left(25^{\circ} \mathrm{C}\right)$, refrigeration temperature $\left(4^{\circ} \mathrm{C}\right)$, oven temperature $\left(50^{\circ} \mathrm{C}\right)$. The glass beakers were kept undisturbed for 30 days with extra care not to disturb the formed cream layer above the aqueous layer to get the exact stability of emulsion over the storage period of 30 days.

\section{Result and discussion}

\subsection{Effect of mono: dicaprylin on stability of emulsion}

During the study, the effect of the ratio of mono: dicaprylin on emulsion stability the homogenizer pressure was kept constant at $25 \mathrm{Mpa}$. Three combinations of mono: dicaprylin were made i.e., monocaprylin: dicaprylin 30:70, 50:50 and 80:20. It is known that the synergistic effect shows good results as compared to a single emulsifier (Nicholson and Marangoni, 2018). The stability of flavour emulsion during 30 days storage is represented in Figure 1. The percentage stability is calculated using the following expression

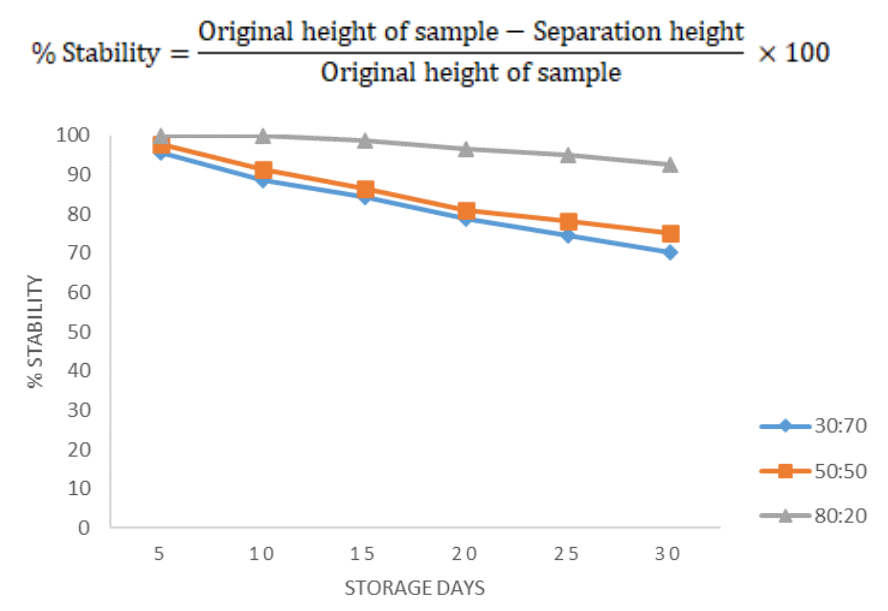

Figure 1. Effect of emulsifier combination on the stability of flavour emulsion 
It is clear from the figure that as the content of monocaprylin increases in the combination of emulsifier the stability of flavour emulsion also increases. For 30:70 combination of mono:dicaprylin there was a slight separation in two layers at day 5 and stability was seen as $95.5 \%$ which slowly decreased with an increase in storage days and was observed as $70.37 \%$ after 30 days. The $\%$ stability increased to $75.18 \%$ for the $50: 50$ combination after 30 days and was $92.59 \%$ for the $80: 20$ combination of mono: dicaprylin. This may be due to the fact that monocaprylin contains two hydroxyl group as compared to dicaprylin which has only one hydroxyl group. Monoglyceride has more HLB value and is more surface-active. Thus, the combination containing a higher ratio of monocaprylin showed higher stability for 30 days, where other emulsifier ratios of mono and dicaprylin showed moderate stability and there was a decrease in the emulsion stability after 20 days.

\subsection{Effect of homogenization passes on Flavour emulsion stability}

The ultra-high-pressure homogenizer is used for the homogenization of flavour emulsion with a pressure of $25 \mathrm{MPa}$. The effect of homogenizer passes on storage stability of flavour emulsion is shown in Figure 2. The passes used for homogenization of flavour emulsion were $1,2,3,4$. The effect of number of passes was studied keeping emulsifier combination constant as 80:20 (mono: dicaprylin). It is observed that when mixture of flavour emulsion was passed twice from the highpressure homogenizer it showed good storage stability. The flavour emulsion showed $99.2 \%, 95.5 \%, 91.85 \%$ stability after 10, 20 and 30 days of storage respectively. From Figure 2 it is observed when mixture of flavour emulsion was given three and four passes there was only marginal increase in the stability of flavour emulsion. Three passes showed $99.6 \%, 95.5 \%, 92.2 \%$ stability after 10,20 and 30 days of storage which was $100 \%, 95.5 \%$,

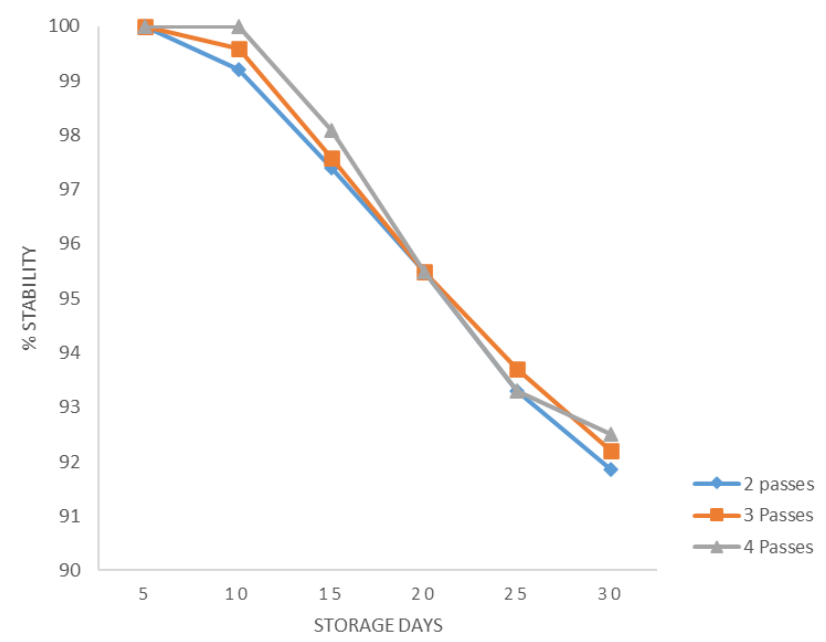

Figure 2. Effect of homogenization passes on flavour emulsion stability
$92.5 \%$ for sample which was given four passes in homogenizer. The results obtained revealed that with increase in the homogenization passes there is only significant increase in the stability of flavour emulsion. But keeping in mind the economy of the process on large scale, the optimum homogenization pass for good storage stability of flavour emulsion was optimized as 2 passes in the present study. The homogenization passes varies greatly with the type and amount of oil phase used for making of flavour emulsion.

\subsection{Viscosity}

Viscosity is resistance to flow. The viscosity of flavour emulsion containing 80:20 combination of monoglyceride: diglyceride was noted before homogenization and after homogenization process which is represented in Figure 3. From the figure, it is observed that homogenization process has not affected the viscosity of the flavour emulsion. Figure 3 shows a linear relationship between shear stress and shear strain. Hence, the flavour emulsion behaves like a Newtonian fluid before and after homogenization process. There is no shear thinning or shear thickening observed. Such flavour emulsion can find wide application in beverages, dairy, bakery and confectionery industries. From the figure, it is observed that the plot between shear rate and shear stress shows a linear relationship. According to Newton's law of viscosity, any fluid showing linear behaviour are Newtonian fluids. Hence, flavours emulsion is Newtonian fluid before and after the homogenization process. The expression for newton's law of viscosity is given below (Franco and Partal, 2000).

$$
\tau=-\mu \frac{d u}{d y}
$$

Where $\tau=$ Shear stress (MPa), $\mu=$ Viscosity, $\frac{d u}{d y}=$ Shear rate $\left(\mathrm{s}^{-1}\right)$.

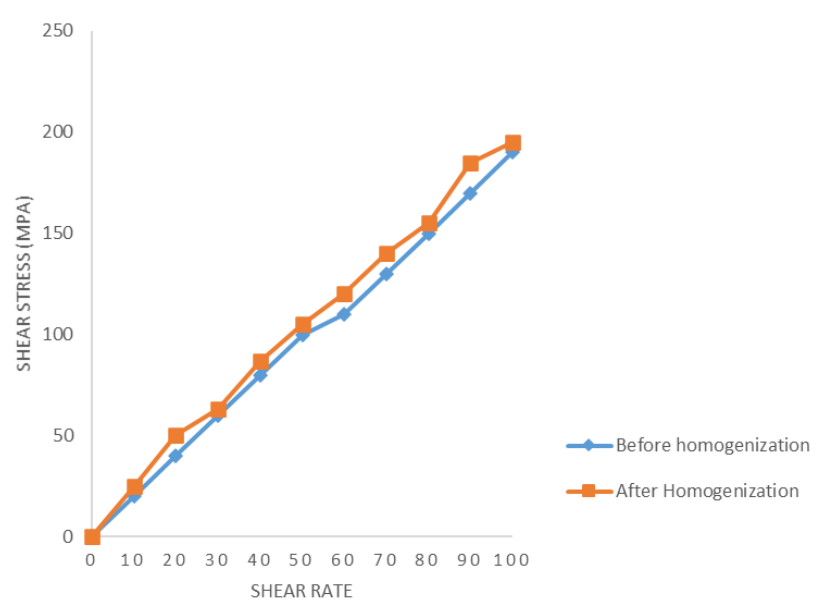

Figure 3. The viscosity of flavour emulsion before and after homogenization

It is very likely that the flavour emulsion shows Newtonian behaviour since the amount of water used for 
the formulation of flavour emulsion was higher as compared with the other ingredients. The use of ultrahigh-pressure homogenizer has a negligible effect on the viscosity of the resulting flavour emulsion. Since the process did not affect the viscosity of the flavour emulsion its texture and flow properties were very well maintained. The flavour emulsion was able to flow easily before and after the homogenization process and has not shown any characteristics of shear thinning and shear thickenin.

\section{$3.4 \mathrm{pH}$}

The $\mathrm{pH}$ was measured using a $\mathrm{pH}$ meter. The $\mathrm{pH}$ of all the three combinations of emulsifier was found to be between $6.5-6.9$. The use of monocaprylin and dicaprylin as an emulsifier has no effect on the $\mathrm{pH}$ of flavour emulsion. This is because the monocaprylin and dicaprylin are non-ionic emulsifiers and hence they did not have any notable effect on the $\mathrm{pH}$ of flavour emulsion. Thus, the $\mathrm{pH}$ of the flavour emulsion remained very close to the neutral scale.

\subsection{Creaming index}

The creaming index of flavour emulsion is calculated using the following expression (Loi et al., 2019).

$$
\text { Creaming Index }=100 \times \mathrm{H}_{\mathrm{S}} / \mathrm{H}_{\mathrm{E}}
$$

Where $\mathrm{CI}=$ Creaming Index, $\mathrm{H}_{\mathrm{S}}=$ Height of cream and $\mathrm{H}_{\mathrm{E}}=$ Total height of Emulsion

The creaming index of flavour emulsion obtained from the combination of emulsifier monocaprylin: Dicaprylin with the ratio of 80:20 was evaluated. It is shown in Figure 4. From the figure, it is seen that in the initial storage period the creaming index is observed to be zero. The creaming index slowly increases which means there is a separation of oil phase from the continuous phase, but the effect is negligible. After 30 days, the flavour emulsion was quite stable. The

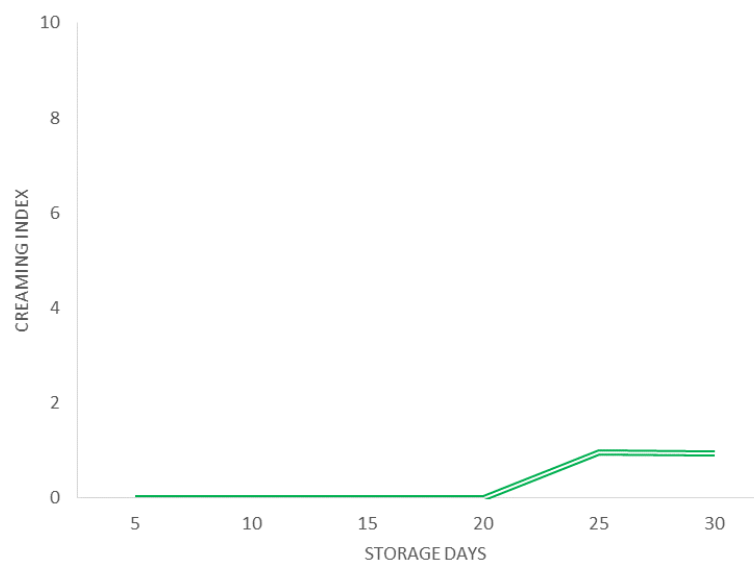

Figure 4. Creaming index of flavour emulsion made by 80:20 combination of mono: dicaprylin creaming index should be zero for a good emulsion (McClements, 2007). However, the flavour emulsion showed a slow increase in the creaming index over a storage period of 30 days. For the first few days, the creaming index was zero for the flavour emulsion. Then gradually, the creaming index increased to 0.0033 , $0.0055,0.96$, and 0.94 after $15,20,25,30$ days respectively. The obtained result showed that a higher ratio of monocaprylin in the emulsifier combination has an excellent effect on the stability of the emulsion, hence they can be effectively utilized in various food and related applications.

\subsection{Stability study}

Stability study of flavour emulsion made from emulsifier with the combination of monocaprylin: dicaprylin at ratio 80:20 was studied at three different conditions viz ambient temperature $\left(25^{\circ} \mathrm{C}\right)$, high temperature $\left(50^{\circ} \mathrm{C}\right)$ and refrigerator temperature $\left(4^{\circ} \mathrm{C}\right)$. The effect of emulsifier and homogenization on flavour emulsion at different storage temperature is shown in Figure 5. It was investigated that the flavour emulsion was more stable at room temperature and cold temperature. A cold temperature study was done by keeping the sample in the refrigerator at $4^{\circ} \mathrm{C}$, whereas high temperature study was done by keeping the sample in the oven at $50^{\circ} \mathrm{C}$. The flavour emulsion was found to be less stable at oven temperature as compared to cold and room temperature. Since flavour compounds are volatile and less stable at high temperature, which decreases their stability at high temperature. But the flavour compounds are stable at ambient temperature and refrigeration temperature. The flavour emulsion stored at room temperature showed higher stability of $100 \%$ up to 10 days after that there was a slow decline instability as $98.8 \%, 96.6 \%, 95.18 \%$ and $92.59 \%$ after $15,20,25,30$ days respectively. The same trend was observed for flavour emulsion stored at $4^{\circ} \mathrm{C}$, it also showed $100 \%$ stability for 10 days which gradually decreases to $96.6 \%$, $93.7 \%, 91.8 \%, 89.25 \%$ at $15,20,25,30$ days

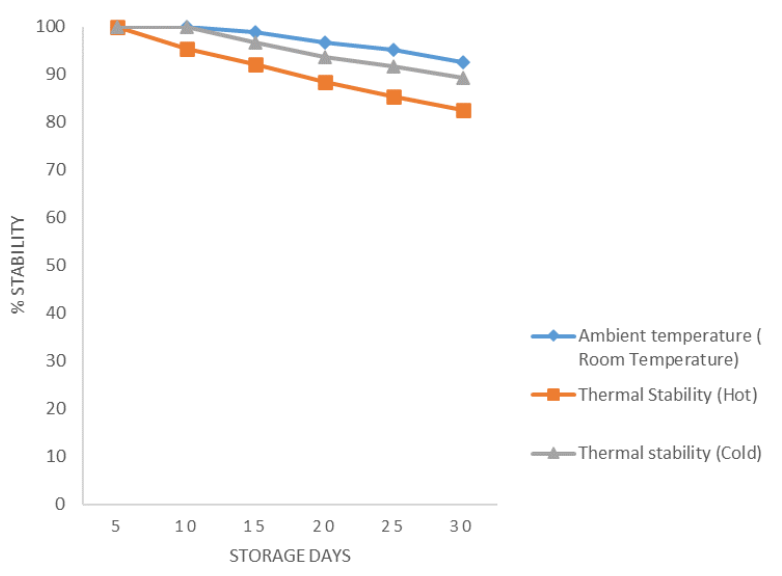

Figure 3. The viscosity of flavour emulsion before and after homogenization 
respectively. But the flavour emulsion sample stored at $50^{\circ} \mathrm{C}$ showed $100 \%$ stability only up to 5 days and then the stability decreased at a faster rate with the stability of less than $82 \%$ after 30 days of storage.

\section{Conclusion}

The present study evaluates the effect of different monocaprylin and dicaprylin combination on the stability of flavour emulsion. It is investigated that high monocaprylin content was effective in achieving the lowest creaming index with stable flavour emulsion for 30 days of storage time. Further, the application of nonthermal technique like ultra-high-pressure homogenization also had an excellent effect on the stability of flavour emulsion without effecting the rheological characteristics of flavour emulsion. The flavour emulsion exhibited Newtonian characteristics before and after the homogenization process at $25 \mathrm{MPa}$ and two passes. The flavour emulsion with higher monocaprylin content showed zero creaming index for 10 days which came down to 0.94 after 30 days which is negligible. The flavour emulsion showed higher stability at ambient temperature $\left(25^{\circ} \mathrm{C}\right)$ and refrigeration temperature $\left(4^{\circ} \mathrm{C}\right)$ making it more suitable for dairy and beverages applications. The present result is useful in developing food products using flavour emulsion that allows the flavours in processed foods to remain stable on the shelf for a longer period of time, thus increasing the acceptability of the processed foods.

\section{Conflict of interest}

The authors confirm that they have no conflicts of interest with respect to the work described in this manuscript.

\section{Acknowledgement}

The author is thankful to the Department of Science and Technology, Government of India for providing DST- Inspire fellowship for doing doctoral research.

\section{References}

Baer, R.J., Wolkow, M.D. and Kasperson, K.M. (1997). Effect of Emulsifiers on the Body and Texture of Low Fat Ice Cream. Journal of Dairy Science, 80 (12), 3123-3132. https://doi.org/10.3168/jds.S00220302(97)76283-0

De Oliveira, R.C., Davantel De Barros, S.T. and Gimenes, M.L. (2013). The extraction of passion fruit oil with green solvents. Journal of Food Engineering, 117(4), 458-463. https:// doi.org/10.1016/j.jfoodeng.2012.12.004
Franco, J.M. and Partal, P. (2000). Rheology - Vol. 1 The Newtonian Fluid. Retrieved from Encyclopedia of Life Support Systems (EOLSS).

Hasan, N.M.Y. (2014). Role of medium-chain fatty acids in the emulsification mechanistic of self-microemulsifying lipid formulations. Saudi Pharmaceutical Journal, 22(6), 580-590. https:// doi.org/10.1016/j.jsps.2014.02.005

Loi, C.C., Eyres, G.T. and Birch, E.J. (2019). Effect of mono- and diglycerides on physical properties and stability of a protein-stabilised oil-in-water emulsion. Journal of Food Engineering, 240, 56-64. https:// doi.org/10.1016/j.jfoodeng.2018.07.016

Mazzutti, S., Pedrosa, R.C. and Salvador Ferreira, S.R. (2020). Green processes in Foodomics. Supercritical Fluid Extraction of Bioactives. In Cifuentes, A. (Ed.) Comprehensive Foodomics, p. 725-743. USA: Elsevier. https://doi.org/10.1016/B978-0-08-1005965.22816-3

McClements, D.J. (2007). Critical review of techniques and methodologies for characterization of emulsion stability. Critical Reviews in Food Science and Nutrition, 47(7), 611-649. https:// doi.org/10.1080/10408390701289292

Nicholson, R.A. and Marangoni, A.G. (2019). Diglycerides. In Melton, L., Shahidi, F. and Varelis, P. (Ed.) Encyclopedia of Food Chemistry, p. 70-73. USA: Elsevier. https://doi.org/10.1016/B978-0-08100596-5.22343-3

Norn, V. (2015). Emulsifiers in Food Technology. United Kingdom: Wiley. https:// doi.org/10.1002/9781118921265

Ordóñez-Santos, L.E., Esparza-Estrada, J. and VanegasMahecha, P. (2020). Ultrasound-assisted extraction of total carotenoids from mandarin epicarp and application as natural colorant in bakery products. LWT, 139, 110598. https://doi.org/10.1016/ j.lwt.2020.110598

Osorio-Arias, J.C., Vega-Castro, O. and MartínezMonteagudo, S.I. (2021). 3.15 - Fundamentals of High-Pressure Homogenization of Foods. In Knoerzer, K. and Muthukumarappan, K. (Ed.) Innovative Food Processing Technologies, p. 244 273. USA: Elsevier. https://doi.org/10.1016/B978-008-100596-5.23021-7

Jensen, R.G., Sampugna, J. and Gander, G.W. (1961). Glyceride and Fatty Acid Composition of Some Mono-Diglyceride Ice Cream Emulsifiers. Journal of Dairy Science, 44(6), 1057-1060. https:// doi.org/10.3168/jds.S0022-0302(61)89856-1

Ropkins, K. and Taylor, A.J. (1996). the Isolation of Flavour Compounds from Foods by Enhanced 
Solvent Extraction Methods. In Taylor, A.J. and Mottram, D.S. (Ed.) Flavour Science. Recent Developments. USA: Woodhead Publishing Limited. https://doi.org/10.1533/9781845698232.5.297

Saifullah, M. and Aziz, M. (2012). Development of orange flavour emulsion. Journal of the Bangladesh Agricultural University, 9(2), 291-296. https:// doi.org/10.3329/jbau.v9i2.11043

Shama, F. and Sherman, P. (1973). Identification of stimuli controlling the sensory evaluation of viscosity II. Oral Methods. Journal of Texture Studies, 4(1), 111-118. https://doi.org/10.1111/ j.1745-4603.1973.tb00657.x

Spyropoulos, F., Lloyd, D.M., Hancocks, R.D. and Pawlik, A.K. (2014). Advances in membrane emulsification. Part A: Recent developments in processing aspects and microstructural design approaches. Journal of the Science of Food and Agriculture, 94(4), 613-627. https://doi.org/10.1002/ jsfa. 6444

Wang, Y., Wang, S., Fabroni, S., Feng, S., Rapisarda, P. and Rouseff, R. (2020). Chemistry of citrus flavor. In Talon, M., Caruso and Gmitter, F.G. (Ed.). The Genus Citrus, Vol. 1, p. 447-470. USA: Woodhead Publishing. https://doi.org/10.1016/B978-0-12812163-4.00022-X

Wilde, P.J. (2019). Improving Emulsion Stability Through Selection of Emulsifiers and Stabilizers. In Reference Module in Food Science. Elsevier. https:// doi.org/10.1016/B978-0-08-100596-5.22337-8 